\title{
Diskussion zum Vortrag von \\ Friedrich-Christian Schroeder
}

\author{
Leitung: Oкко Behrends
}

\section{BEHRENDS:}

Vielen Dank für den Vortrag. Wir haben, wenn ich auf die Zeit sehe, eine gute halbe Stunde für die Diskussion. Ich fand besonders eindrucksvoll, dass der Begriff des Rechtsnihilismus schon 1918 geprägt worden ist, der doch ganz neulich erst von MEDWEDEw in der Art einer neuen Einsicht als Haupthindernis auf dem Weg zum Rechtsstaat herausgestellt worden ist. Sehr einleuchtend fand ich auch den Hinweis, dass ein solcher Staat, der vor allen Dingen seine Ziele durchsetzen will, an äußerem Gehorsam interessiert ist und die Gesinnung als etwas Indifferentes ansieht. Ich darf die Diskussion eröffnen, wer möchte das Eis brechen, Herr Diederichsen?

\section{DiEDERICHSEN:}

Ja, Herr Schroeder, auch ich habe Ihnen fasziniert zugehört, weil Ihre Darstellung ein so schönes klares Bild von der Instrumentalisierung des Rechts in der DDR gab. Allerdings haben Sie sich, wie Herr Behrends das eben schon sagte, darauf beschränkt, uns zu zeigen, wie das Gesetz und die Rechtserziehung im Grunde lediglich der Erreichung rein politischer Ziele dienten und dass es in der DDR immer nur um die Erzwingung von Rechtsgehorsam ging. Als wir das Thema in unserer Kommission besprachen, ging es uns in der Tat vor allem um eine solche positivistische Darstellung. Erziehung hat aber, selbst wenn sie in Form von erziehender Gesetzgebung erfolgt, immer auch rechtsübergreifende kulturelle Aspekte; und dafür gibt die DDR wahrscheinlich mehr Beispiele als das folgende, auf das ich mich hier beschränken will.

Ich habe in den 1950er Jahren an der Freien Universität in Berlin studiert, war aber immer wieder auch in Ostberlin in der Humboldt-Universität, wo ich einmal einen Vortrag der damaligen Justizministerin der DDR, Hilde BenjaMIN, gehört habe, die in der Bevölkerung nur die „Rote Hilde“ genannt wurde, weniger weil sie Kommunistin war als wegen ihrer willkürlichen und mit der Guillotine vollstreckten Todesurteile, die sie als oberste Richterin in der Sowjetzone gefällt hatte. In ihrem damaligen Vortrag ging es aber um etwas anderes, nämlich um die Gleichberechtigung der Frauen, und damit um einen Punkt, in welchem sich die DDR im Grundsatz wesentlich „,fortschrittlicher“ gebärdete als die Bundesrepublik. 
In der zweiten Hälfte der 1980er Jahre nutzte Thilo Ramm das auf die Bemühungen von Willy Brandt und Helmut Schmidt zurückgehende deutschdeutsche Kulturabkommen dazu, westdeutsche und ostdeutsche Juristen zusammenzubringen, und zwar im Arbeits- und Familienrecht als den beiden Gebieten, in denen sich die DDR-Führung für besonders kompetent, ja für bahnbrechend hielt. Beschränken wir uns auf das Familienrecht, so hat die DDR - wie die Bundesrepublik in ihrem Grundgesetz - die Gleichberechtigung von Mann und Frau in der Verfassung festgeschrieben, darüber hinaus aber in der Tat auch dafür gesorgt, dass die Gleichberechtigung ökonomisch verstanden wurde, indem der sozialistische Staat sicherstellte, dass Frauen erwerbstätig bleiben konnten, auch wenn sie Kinder zu versorgen hatten. Sie gingen alle einer Arbeit nach und verfügten so über eigenes Einkommen und hatten auf diese Weise die Gleichberechtigung nicht nur als juristischen Grundsatz, sondern auch ökonomisch völlig internalisiert.

Nach der Wende kam dann der Schock. Plötzlich stimmten die Bedingungen für das Gleichberechtigungsgefühl nicht mehr. Mir ist das zum ersten Mal klar geworden, als in dem im Rahmen des deutsch-deutschen Kulturabkommens gebildeten und nach 1990 fortgesetzten Arbeitskreis „Familienrecht“ jemand aus der DDR beiläufig bemerkte, die Frauen hätten ja in „geschützten Brigaden“ gearbeitet. Auf unsere Frage, was darunter zu verstehen sei, kam zögernd heraus, dass die Frauen vielfach gar nicht richtig in den eigentlichen Produktionsprozess eingebunden gewesen seien. Vielmehr wären sie in eigenen Abteilungen zusammengefasst worden, hätten während der Arbeitszeit auch einkaufen gehen können, etwa wenn es darum ging, für seltene Waren anstehen zu müssen; und hätten auch, wenn die Kinder krank waren, zu Hause bleiben dürfen, was in einem wirklich durchgängig ökonomisierten Betrieb betriebswirtschaftlich gar nicht möglich gewesen wäre. Verpuffte damit nicht ein großer Teil der Erziehung zur ökonomischen Gleichberechtigung? Erwies sich nicht auch die wirtschaftliche Eigenständigkeit der Frauen spätestens mit der Auflösung ihrer nicht produktiven Arbeitsverhältnisse beim wirtschaftlichen Zusammenbruch des sozialistischen Regimes als Illusion? Oder - um die Problematik durch eine entsprechende Erweiterung zu verallgemeinern: Wie steht es eigentlich mit der Effektivität anderer erziehender Gesetze sozialistischer Staaten wie der DDR?

Schroeder:

Ja, das sind natürlich sehr interessante persönliche Erlebnisse, die Sie da hatten. Ich habe selber auch in Berlin studiert und auch noch zu der Zeit, als es keine Mauer gab und man rüberfahren und viel diskutieren konnte. Aber ganz konsistent fand ich Ihre Darlegung nicht. Sie sagten, die Frauen hatten eigentlich keine 
ökonomische Tätigkeit, denn sie kochten und unterhielten sich und konnten einkaufen gehen usw.

\section{DiederichSEN:}

Kochen habe ich nicht gesagt, aber die Freistellung im Betrieb zum Zwecke des Einkaufens war nötig, damit die Familien überhaupt an die Lebensmittel herankamen, die - weil sie Devisen kosteten - in der DDR knapp waren. Da die Männer in die Betriebsabläufe eingebunden waren, gingen die Frauen und stellten sich an; und so waren es auch sie, die zu Hause bleiben mussten, wenn die Kinder krank waren.

\section{SCHROEDER:}

Ja, aber hatten Sie nicht gesagt, sie übten eigentlich keine ökonomische Funktion aus?

DiEDERICHSEN:

Ich würde es etwas anders ausdrücken: Es war nicht eingeplant, dass mit der Arbeit von geschützten Brigaden Gewinn erzielt wurde.

SCHROEDER:

Ja, aber woher kommt dann das Selbstbewusstsein der Frauen?

\section{DiederichSEN:}

Das gerade wollte ich gerne geklärt wissen. Ich denke, dass der erzieherische Effekt rein politisch war und dabei weder die Belange der Wirtschaft noch die Frauen wirklich ernst genommen wurden.

\section{SCHROEDER:}

Also ich würde das etwas anders sehen. In der Sowjetunion kam in den letzten Jahren zum Vorschein, dass ANDropow Kontrolleure in die Friseurläden und Badeanstalten usw. geschickt hatte, wo sich herausstellte, dass große Teile der Belegschaften blau machten. Und es war ja auch nach der Wende ein bekannter Witz im Westen, dass die Arbeiter, die aus der DDR herübergekommen waren, sich erst einmal hinsetzten und sagten ,Wir warten auf das Baumaterial“, was aber längst da war. Da hat sich einfach ein gewisser Müßiggang als sozialer Besitzstand entwickelt. Und die Leute haben auf diesen sozialen Besitzstand gepocht, aber einen Erziehungserfolg kann ich darin eigentlich nicht sehen. Natürlich hatten die Frauen ein stärkeres Selbstbewusstsein in der DDR, aber das resultierte eben nicht daraus, sondern das hat in der Sowjetunion angefangen, dass die Sowjetunion aus Arbeitskräftemangel in großem Umfang Frauen in das Berufsleben eingespannt hat. Das hat übrigens trotz allen Kinderkrippen usw. zu einem rasanten Bevölkerungsschwund geführt. Diese Doppelbelastung ist eben für Frauen schwer erträglich, aber ich will jetzt nicht mit Frau Schumann in eine 
Auseinandersetzung über die Stellung der Frau in der modernen deutschen Gesellschaft kommen. Aber die Arbeitsteilung - Mann berufstätig, Frau Haushalt - hatte natürlich auch ihren Sinn. Und wenn man bedenkt, was heute Haushalte für ein Knowhow, für ein technisches Wissen, für eine Organisationstätigkeit erfordern, dann ist das schon fast ein Fulltime-Job. Also meiner Meinung nach hat man es nicht nötig, eine Arbeitsstelle zu finden, um Selbstbewusstsein zu bekommen.

\section{BEHRENDS:}

Herr Stolleis bitte.

\section{Stolleis:}

Herr Schroeder, ich habe in den letzten Jahren mich intensiver mit der Wissenschaftsgeschichte des öffentlichen Rechts in der DDR beschäftigt ${ }^{\star}$ und ich möchte vorweg sagen, dass ich im Bereich des Verwaltungs- und Staatsrechts in den wissenschaftlichen Arbeiten relativ wenig von diesem edukatorischen Element gespürt habe - dagegen sehr viel bei der Strafrechtspraxis aufgrund der Gesetze, also bei der Erziehung durch Gesetz und Richterspruch. Wenn man etwa nachliest, was Inga MARKovits in ihrem Buch „Gerechtigkeit in Lüritz“ (2006) beschreibt, dann ist die Funktion des Richters doch sehr stark erzieherisch, viel stärker als bei uns, etwa gegen Bummelantentum, Kleindiebstahl von Volkseigentum, Alkoholmissbrauch, Ehebrüche und alles dies. Die Erziehungsfunktion steht deutlich vor der Straffunktion und vor der Anbindung an das strenge Gesetz. Wenn man noch das Sammelsurium von Orden und Auszeichnungen dazu nimmt, was Sie ja auch berührt haben, dann sieht man, dass diese Staaten doch ganz stark auf Aktivierung, auf Kämpfertum und auf eine Ethisierung des täglichen Lebens hinarbeiten, wahrscheinlich zur Kompensation von ökonomischen Mängeln und Alltagsschwierigkeiten, die durch diese Ethisierungen überbrückt werden sollen. Wenn eine Ehe zu Bruch ging, wurden die Leute einbestellt und erst einmal im Betrieb selbst ermahnt, ob es nicht doch geht, und wenn es zur Ehescheidung kam, musste noch einmal ein erzieherisches Vorgespräch geführt werden. Dieser starke erzieherische Impetus ist nicht einfach zu erklären. Jedenfalls vermischen sich ideologische und ökonomische Motive.

SCHROEDER:

Sie haben ja gemerkt, dass ich dieser Verwendung des Begriffs „erzieherisch“ und „edukatorisch“" sehr vorsichtig gegenüberstehe. Ist nicht das, was Sie schildern, dass das Ehepaar in den Betrieb einbestellt wird und dass der Richter mit dem Angeklagten diskutiert, eher etwas, was wir heute unter Mediation verstehen?

^ Michael Stolleis, Sozialistische Gesetzlichkeit. Staats- und Verwaltungsrechtswissenschaft in der DDR, 2009. 
Das waren doch keine moralischen Sachen, die sie da mit nach Hause bekamen, sondern es wurde versucht, durch Mediation diese Konflikte auszugleichen. Und wenn Sie jetzt sagen, der Richter hatte eine sehr viel stärkere moralische Funktion, dann hätte der Richter allein das ja gar nicht machen können, sondern er konnte das nur, weil ihm kollektive Instrumente zur Seite standen, also die Konfliktkommissionen in den Betrieben. Dann gab es ja auch das Kollektiv, also die Betriebsbelegschaft, als Bewährungshelfer. Es wurden Betriebsvertreter, gesellschaftliche Ankläger und Verteidiger zu den Strafverhandlungen geladen, die dort den Leumund des Betriebes darlegten, also den üblen Leumund oder den guten Leumund. Das war meiner Meinung nach durch einen starken kollektiven Druck erzeugt und kann nicht unbedingt der Rechtspflege der DDR zugute gehalten werden.

\section{Sellert:}

Herr Schroeder, vielen Dank für Ihre Ausführungen und Ergebnisse, die zu einem Vergleich mit Erziehungsgedanken im NS-Staat, aber auch mit solchen in der Volksrepublik China anregen. Dankbar bin ich für Ihre scharfsinnige Unterscheidung zwischen Erziehung durch Gesetz und zum Gesetz. Ich habe den Eindruck, dass Sie darunter zwei verschiedene Kategorien verstehen. Müsste man oder könnte man nicht auch sagen „Erziehung durch Gesetz zum Gesetz“, denn wie soll man zum Gesetz erziehen, wenn nicht durch Gesetz? Das gilt auch für den Richter, der das Gesetz anwendet und erzieherisch tätig wird, indem er beispielsweise durch die im Gesetz vorgesehenen Sanktionen den jugendlichen Straftäter zu einem gesetzmäßigen Verhalten veranlassen will. Insoweit überzeugt mich Ihre Differenzierung nicht in jeder Hinsicht.

Hinweisen möchte ich noch darauf, dass nach meiner Kenntnis die positive Spezialprävention, also die Resozialisierung, nicht erst um 1960 eine Rolle zu spielen begann. Sie hat vielmehr, soweit es sich um den Besserungsgedanken handelt, schon im Strafvollzug der Zuchthäuser von Bridewell, Amsterdam, Bremen, Celle etc. eine Rolle gespielt, war lebhafter Diskussionsgegenstand im 19. Jahrhundert, wo es beispielsweise CARL TheOdor Welcker oder Franz von LiszT für die Strafe um die innere oder moralischen Besserung des Täters ging.

\section{SCHROEdER:}

Ich möchte beiden Sachen etwas entgegenhalten, wenn Sie erlauben. Also einmal die Erziehung zum Gesetz. Da können Sie natürlich sagen, das sei eigentlich dasselbe, die Erziehung durch Gesetz und die Erziehung zum Gesetz. Sie haben dann diese schöne Kette gebildet: durch das Gesetz zum Gesetz. Aber ich verstehe unter Erziehung durch das Gesetz mehr als die bloße Erziehung zur Einhaltung gesetzlicher Vorschriften, sondern die Herausbildung von Werten, einer eigenen Wertdisposition, so dass das nicht aus Beachtung des Gesetzes, 
sondern aus eigenem inneren Antrieb erfolgt. Und danach müssten wir, finde ich, bei unserer Tagung „Erziehung durch Gesetz“ suchen. Ob es so etwas gibt. Und wenn Sie jetzt mit der Besserung ankommen, das ist ja nun eine Frage der Spezialprävention durch den Strafvollzug, das sind wieder die gesetzlich angeordneten Folgen. Aber die positive Generalprävention bedeutet gerade, dass die Strafdrohung als solche dazu führt, dass die in dem Strafgesetz enthaltenen Verbote dadurch wirksam werden, dass in den - sagen wir mal - Konsumenten des Strafgesetzes oder den Lesern des Strafgesetzes ein Wertbewusstsein, ein positives Wertbewusstsein, entsteht. Das ist ja gerade der eigentliche Ansatz der positiven Generalprävention, nicht mehr Abschreckung und nicht mehr bloß Beachtung des Gesetzes, sondern die Erzeugung von Werten.

RÜCKERT:

Das ist Erziehung durch das Gesetz.

\section{BEHRENDS:}

Herr Avenarius bitte.

Avenarius:

Sie haben am Anfang hingewiesen auf diese kurze Phase 1918, in der man glaubte, völlig auf das Recht verzichten zu können, haben dann gesagt, man habe an die Stelle des Rechts das sozialistische Rechtsbewusstsein setzen wollen, und schließlich gesagt, die Grundlage für den Gedanken, das sozialistische Rechtsbewusstsein trete an die Stelle des gesetzten Rechts, sei die Psychologische Rechtslehre von PeTrAżyCKI gewesen, wenn ich es richtig verstanden habe. Ich habe mir das immer anders vorgestellt, und vielleicht lohnt es sich, darauf hinzuweisen, weil der Gedanke der Erziehung durch Recht bei PeTrażycKi ja so wichtig ist. Er sagt doch, dass die wünschenswerte ethische Einstellung des Menschen die berühmte imperativ-attributive Grundlage voraussetze, und diese sei das Recht. Das ist es, wogegen Tolstoj dann so hart polemisiert. Er sagt also: Das Recht brauche ich, es geht nicht, dass sich die entsprechende ethische Einstellung nur aus dem Menschen selbst entwickelt. Ich meine nun, dass diese Lehre durchaus zum Zug kommt, aber erst etwas später, nämlich vermittelt womöglich durch Petrażyckis Schüler RejSNer in der Situation, als man nach dem Kriegskommunismus sagt: Vorläufig brauchen wir ein Recht - natürlich im Lichte des sozialistischen Rechtsbewusstseins, das dann Eingang in das Recht findet durch wertausfüllungsbedürftige Rechtsbegriffe.

SCHROEDER:

Ja, das war eine Ergänzung, gegen die ich nichts einzuwenden habe, sondern für die ich danke. 
BEHRENDS:

Wunderbar. Frau Wendehorst bitte.

WENDEHORST:

Ja, herzlichen Dank. Ich möchte noch einmal in eine ähnliche Kerbe schlagen wie Herr Sellert, nur anders. Ich denke auf jeden Fall, dass wir differenzieren müssen zwischen einerseits Erziehung durch Gesetz bzw. Verhaltenssteuerung durch Anreize, sei es positiver oder negativer Art, und Bewusstseinsbildung andererseits. Ich denke nur nicht, dass wir so scharf differenzieren müssen und vor allem nicht, dass wir es als Gegensatz konstruieren dürfen. Ich will einmal ein Beispiel bringen: Wenn der Gesetzgeber einen Mordtatbestand in das Gesetz schreibt, dann will er natürlich damit auch das Bewusstsein vermitteln, dass man so etwas nicht tut. Das Problem ist nur, dass es so viele und vielleicht vordringliche andere Gründe gibt, den Mord zu verbieten und unter Strafe zu stellen, dass der Erziehungseffekt ein bisschen vertuscht, verdeckt wird, und dass wir natürlich, wie Sie es auch sehr schön formuliert haben, vom Hundertsten ins Tausendste kommen würden, wenn wir jetzt den Mordtatbestand und den Diebstahlstatbestand auf ihre edukatorischen Effekte hin untersuchen würden. Aber sie sind doch immer da.

\section{SCHROEDER:}

Ja, aber erkannt und diskutiert wurde diese Funktion erst durch die Lehre von der Frankfurter Schule, durch die in den 1960er Jahren entwickelte Lehre von der positiven Generalprävention. Davor hat man sich immer mit der Abschreckung beschäftigt; immer hieß es: Abschreckung ist psychologischer Zwang, siehe Feuerbach, und das ist die rechtsstaatliche Wirkung des Strafrechts, dass man den potentiellen Täter von vornherein durch Drohungen davon abhalten möchte, überhaupt Straftaten zu begehen. Feuerbach hatte ja dieses schöne Bild von dem Kräfteparallelogramm, die Strafdrohung muss gerade so stark sein wie der Anreiz zur Straftat ist, dann entsteht ein Gleichgewicht und es kann gar nicht zu Straftaten kommen. Und das, was Sie sagen: Natürlich will der Gesetzgeber auch mal das andere, aber in den strafrechtlichen und straftheoretischen Büchern ist das lange Zeit nicht angesprochen worden.

BEHRENDS:

Ich habe mich gerade auf die Liste gesetzt und hätte eine Frage im Hinblick auf die Wirkung von Gesetzen. Wirken Gesetze eigentlich nicht immer erst auf der institutionalisierten Ebene, wo man sie als Einrichtungen entweder positiver oder negativer Art erlebt? Negativ z.B. nach der Abschaffung des Erbrechts in der Sowjetunion: Sie bedeutete, dass eine gewisse Zukunftssicherheit nicht mehr gegeben war, und man behauptet, dass dort die Leute angefangen haben, 
ihr restliches Vermögen zu vertrinken, weil es sinnlos geworden war, es zu erhalten. Sie erlebten in dieser Ordnung Freiräume, die man erzieherisch nicht als nützlich ansehen kann. Aber führt das nicht zu der Feststellung, dass die Gesetze erziehen, je nachdem wie die auf ihrer Grundlage entstandenen Freiräume oder umgekehrt die Begrenzungen der Handlungsräume erlebt werden? Ihr Beispiel der betrieblichen Mediation hat insofern sehr anschaulich vor Augen gerückt, mit welchem sozialen Druck solche institutionalisierten Begrenzungen der Freiräume ausgestattet werden können. Das dahinter stehende Gesetz verblasst demgegenüber. Die Gesetze als bloße Präskription in der oberen Abstraktionsebene sind also vielleicht nicht das, was man betrachten muss, wenn man nach ihrer erzieherischen Wirkung sucht, sondern mehr das institutionell Realisierte und deswegen vom Normadressaten als verhaltensrelevante Ordnung Wahrgenommene. Ich orientiere mich an dem, was verwirklicht ist. Das ist meine Frage.

\section{SCHROEDER:}

$\mathrm{Zu}$ Ihrem Hinweis auf die Abschaffung des Erbrechts noch eine kurze rechtshistorische, etwas witzige Ergänzung. Die Abschaffung des Erbrechts in Russland hat nicht dazu geführt, dass die Leute ihr Vermögen versoffen, sondern dass sie es verschenkt haben. Und da merkte der Gesetzgeber: Um Gottes willen, wenn ich das Vererben verbiete, dann muss ich aber auch die Schenkung verbieten, so wie bei uns im Erbschaftsteuergesetz, da ist ja auch die Schenkung geregelt. Und deswegen ist ein paar Monate später schnellstens hinterhergeschickt worden, dass Verschenken ebenfalls unzulässig ist. Aber zu dem anderen: Wenn Sie sagen: „Ich brauche die Institution“, ist das nicht eigentlich der Hinweis auf ein erfor-

derliches Justizsystem, oder sehen Sie noch etwas dazwischen, zwischen Gesetz und Bewusstsein? Institution, das ist doch eigentlich die Justiz.

BeHRENDS:

Ich meine die gesamten vom Gesetz erlebbar veränderten Lebensumstände. Die Justiz ist hier nur ein - allerdings nicht unwichtiger - Faktor. Wir fahren dann fort, wir haben jetzt noch sechs Wortmeldungen. Ich würde vorschlagen, im Interesse der Zeit zu bündeln, jeweils drei, Herr Heun, Herr Starck und Herr Eichenhofer. Also Herr Heun als Erster.

Heun:

Wenn man Ihrem Vortrag lauscht, fällt auf, dass jedenfalls der Erziehungsgedanke bei aller Differenzierung sehr viel häufiger in der Rechtstheorie dort betont wird als bei uns. Und dann stellt sich natürlich die Frage, was ist der Hintergrund dafür. Jetzt wollte ich Sie zu Erklärungsversuchen befragen. Erstens: Ist das vielleicht auch eine Kompensation dafür, dass das Recht insgesamt weniger Verbindlichkeit hat und beansprucht, dass es durch politische Überlegungen zur 
Seite gedrängt werden kann, so dass auch der Erziehungsgedanke natürlich sehr viel besser mit politischen Überlegungen verbunden werden kann und von daher die strikte Verbindlichkeit des Rechtes einfach nicht denselben Status hat wie bei uns? Zweitens: Kommt da nicht auch zum Ausdruck der sehr unterschiedliche Zugang zum Recht? Bei uns dient Recht in erster Linie auch dem Individualrechtsschutz. Diese Funktion hat das Recht dort gar nicht, sondern es erinnert sehr viel mehr an die Zeit des 16.-18. Jahrhunderts, indem nämlich das Recht auch der Beeinflussung des Kollektivs, der Gesamtheit gilt, also die Perspektive widerspiegelt, dass der Staat über die Herrschaftsträger den Bürger, der im Wesentlichen als reines Objekt hier auftaucht und erscheint, beeinflussen will. Drittens: Müsste man - das hat schon Herr Stolleis angedeutet - nicht noch stärker nach der Bedeutung des Rechts in dem jeweiligen Gebiet differenzieren? Die Funktion auch der Verbindlichkeit, aber auch die Erziehungsfunktion ist natürlich sehr viel stärker im Strafrecht, wo der Staat oder die Herrschaftsträger die Herrschaftsunterworfenen beeinflussen wollen. Sie haben immer nur allgemein über die Funktion des Rechts gesprochen, aber müsste man in dieser Hinsicht doch nicht noch sehr viel stärker differenzieren zwischen den Bereichen des Strafrechts einerseits, des Familien- und Zivilrechts andererseits und dann drittens des öffentlichen Rechts, wo es im Zweifel am schwächsten ist? Und eine letzte Frage: Kann man vielleicht auch irgendetwas dazu sagen, wie sich das eigentlich praktisch auswirkt angesichts der Tatsache, dass ohnehin die Verbindlichkeit des Rechts eine geringere ist? Gibt es eigentlich schon Rechtstatsachenforschung in dieser Hinsicht? Inwieweit haben also die Bürger das Recht überhaupt in diesen Erziehungsfunktionen wahrgenommen - oder ist ohnehin nicht nur hauptsächlich das, was ideologisch gesagt wird, und weniger das, was im Gesetz steht, maßgebend?

\section{BeHRENDS:}

Ich glaube, es ist vertretbar, dass wir jetzt noch zwei weitere Fragen annehmen; Herr Starck bitte.

\section{StARCK:}

Meine Frage geht in eine ganz andere Richtung als die bisherigen Fragen. Was war vor der Revolution? Es ist ja so, dass Revolutionen, so radikal sie aussehen, immer auf Strukturen zurückgreifen, die vorher waren. Und da ist meine Frage: Welche Rolle hat die byzantinische Kirche, also die russische Kirche gespielt? Das Evangelium war wichtig, der Glaube war wichtig, die Liturgie war wichtig, aber eine Rechtskirche wie im Westen gab es nicht. Und ist vielleicht von daher zu verstehen, dass man glaubte, man kann ganz ohne Recht auskommen; denn es ist merkwürdig, was Sie da geschildert haben, dass man sagt, die Rechtsord- 
nung wird völlig abgeschafft. Ist vielleicht dahinter diese Vorstellung, die in der russischen Kirche herrschte, dass man eigentlich gar kein Recht braucht?

\section{EICHENHOFER:}

Da wollte ich anschließen: Der russische Staat war im Zarenreich nicht durch das Recht zusammengehalten worden, sondern durch andere Mechanismen. Und auch die sozialistische Gesetzlichkeit ist eigentlich niemals wirkmächtig geworden. Es gab damals dort einen relativ schmalen Rechtsstab - nur ein paar Richter. Sie haben im Wesentlichen Republikflüchtige verurteilt. Die Juristen, die ausgebildet wurden, waren im Geheimdienst tätig. Anwälte gab es wenige. Das erziehende Recht kommt in der Gesellschaft nicht vor. Wenn es Konflikte gab, ging man zu den Konfliktkommissionen. Darin saßen verdiente Werktätige, die nach nicht-rechtlichen Maßstäben Krisenmanagement - Social engineering - betrieben. Also wie erklären Sie sich diesen Widerspruch einer Geschichtslosigkeit des Rechts und der fehlenden gesellschaftlichen Wirkmächtigkeit des Rechts in der sozialistischen Gesellschaft?

\section{BEHRENDS:}

Eine hochinteressante Frage.

\section{SCHROEDER:}

Ja, Herr Starck, Sie haben meinen Vorgänger mit der Frage nach der spanischen Inquisition in Verlegenheit gebracht, jetzt kommen Sie mir mit der byzantinischen Kirche. Ich bin da natürlich kein Spezialist, aber Tatsache ist doch, dass tatsächlich in Russland vor der Revolution eine ausgesprochen verbreitete rechtsnihilistische Stimmung herrschte, etwa BAKUnIN und auch die Dämonen von Dostojewski und eine echte Alternative vertrat auch Tolstor. Der propagierte die Ersetzung des Rechts durch Liebe. Das waren alles Saaten, die vorher gesät waren und deswegen in der Bevölkerung - wie gesagt - eine Art Rechtsnihilismus herbeigeführt hatten, wenn nicht sogar Vorbehalte gegenüber dem Rechtsstaat. Darauf haben die aufgebaut, das kann man nicht bestreiten.

Ihre erste Beobachtung ist sicher richtig, dass der Begriff „Erziehung“ im sowjetischen Recht sehr viel häufiger auftaucht als bei uns - in seinen ganzen Variationen, Rechtserziehung, Erziehung zum Gesetz, Rechtsbewusstsein usw. Aber das Ganze tritt eigentlich erst auf nach der Stalinschen Kreierung der neuen Staatsfunktion der erzieherischen Tätigkeit des Staates. Das schlug ein wie eine Bombe, denn bisher bestand ja die Auffassung, dass der Überbau von der Basis gespiegelt wird, daher muss man die Basis umformen, damit der Überbau sich wandelt. Jetzt kam die Idee der aktiven erzieherischen Rolle des Staates. Dadurch kamen die ganzen Diskussionen auf, die von der zentralen Ideologie ausgingen, sich dann auf die Staats- und Rechtstheorie ausweiteten und meines 
Erachtens in den unteren Bereichen nicht mehr genuin ausgebildet wurden. Und wie gesagt das andere, das mehr unter den Begriff der Mediation fällt, die ja auch bei uns jetzt sehr stark im Kommen ist, da muss man wohl sagen, da waren uns die sozialistischen Staaten voraus. Denn dieses Kameradschaftsgericht usw., das wird ja allgemein anerkannt, dass das eine sehr stark resozialisierende Wirkung und auch kriminalitätsverhütende Wirkung hatte, teilweise aus Angst, weil sich die Leute dann abends vor der Belegschaft verantworten mussten und alle hörten $\mathrm{zu}$, andererseits aber auch weil friedliche Lösungsmittel angeboten wurden. Sie sagten, glaube ich, dass dem Recht die Verbindlichkeit gefehlt hat und dass deswegen die Erziehungsfunktion nicht zur Geltung kommen konnte oder gerade zur Geltung kommen sollte? Das habe ich jetzt nicht mehr genau in Erinnerung.

Heun:

Ich hatte gesagt, dass der Erziehungsgedanke auch eine Kompensation zum Teil dafür sein könnte, dass die Verbindlichkeit eben auch unter dem Vorrang der Politik stand, während man im Rechtsstaat allein auf die Verbindlichkeit des Rechts vertrauen kann.

\section{SCHROEDER:}

Von oben wurde natürlich die Verbindlichkeit des Rechts behauptet, auch wenn in der Bevölkerung überhaupt kein Verlass auf das Recht bestand.

BEHRENDS:

Wir müssen fortfahren. Herr Jestaedt bitte.

JeSTAEDT:

Ich würde gerne noch einmal zwei Gesichtspunkte zusammennehmen: nämlich zum einen, was Herr Heun gesagt, und zum anderen, was Frau Wendehorst angesprochen hatte. Die Unterscheidung von Erziehung durch Gesetz und Erziehung zum Gesetz setzt voraus, dass das Gesetz, dass das Recht etwas zweifelsfrei von seiner Umwelt Isolierbares ist. Daran würde ich in unserem Kontext Zweifel hegen und damit auch daran, ob das von uns beackerte Feld der Legaledukation mit der Unterscheidung der Erziehung zum Gesetz und jener durch Gesetz vollständig erfasst werden kann. Sie sprachen von ,positivistisch“. Ich habe groBe Zweifel, ob das der Begriff von Positivismus ist, den wir landläufig einsetzen und einsetzen können. Das Recht würde insoweit ja etwas Selbsttragendes, etwas Autarkes und daher in seinen Wirkungen Isolierbares sein. Das indes scheint mir zweifelhaft zu sein, auch nach dem, was Sie gesagt haben. Recht ist immer auch ein Instrument und in diesem Sinne eine Funktion der Politik, der Moral und so fort. Dementsprechend möchte ich auch Zweifel äußern, ob wir so messerscharf und ganz selektiv die Frage stellen können: Gibt es eine im Einzelnen 
messbare Erziehung durch Gesetz? Gar: Gibt es eine verifizierbare Erziehung durch ein einzelnes Gesetz? Vielleicht ist es doch angebrachter und erkenntniserhellender, einen breiteren Fokus zu wählen. Dann eröffnet sich: Recht ist nur eine von verschiedenen sozialen Funktionen, die in ihren (Wechsel-)Wirkungen berücksichtigt werden müssen. Schon angesichts dessen ist meines Erachtens die Hoffnung nicht allzu groß, die Wirkungen einer einzelnen Funktion belastbar messen und bestimmen zu können.

\section{BEHRENDS:}

Nun noch Herr Rückert bitte.

\section{RÜCKERT:}

Sie hatten Ludwig KNAPP und Petrażycki erwähnt und herangezogen für die Frage eines sozialistischen Rechtsbewusstseins, nach 1918 erst einmal tabula rasa. Frage: Hat man denn KNAPP (,System der Rechtsphilosophie“ von 1857), der ja eigentlich völlig vergessen war, direkt herangezogen in den Quellen von PETRAŻYCKI oder ist das eine Zutat von Ihnen gewesen, eine eigene Verknüpfung? Wenn es direkt herangezogen würde, würde mich interessieren, wie genau. Denn das stimmt eigentlich nicht, KNAPP behauptet nämlich nicht, dass ein Rechtsbewusstsein als solches genüge, damit es juristisch funktioniert.

\section{SCHROEDER:}

Das habe ich irgendwo gelesen, aber daran kann ich mich jetzt nicht mehr erinnern, das müsste man eruieren.

Und was Sie, Herr Jestaedt, sagen, das ist natürlich eine Grundsatzfrage, die richtet sich an uns alle, da möchte ich eigentlich jetzt meine eigene Auffassung nicht geben. Aber ich bin davon ausgegangen: Die letzte Tagung hieß „Das strafende Gesetz" und in Analogie dazu wurde der Titel für diese Tagung gewählt: „Das erziehende Gesetz“. Deswegen habe ich mich daran gehalten und war davon ausgegangen, dass die Aufgabensteller, die Veranstalter, dem Gesetz eine gewisse Funktion zusprachen. Also da müssten Sie Ihre Vorbehalte gegen die Veranstalter richten.

BEHRENDS:

Die Veranstalter nehmen das auf. Ich bedanke mich ganz herzlich für den Vortrag, der sehr viele Einsichten, vor allen Dingen auch in die historische Vergangenheit, eröffnet hat und darf nun alle Diskutanten zum Kaffee bitten. 